\section{Screening the Cucumber Germplasm Collection for Combining Ability for Yield}

\author{
Todd C. Wehner ${ }^{1,2}$ and Nischit V. Shetty ${ }^{2}$ \\ Department of Horticultural Science, North Carolina State University, \\ Raleigh, NC 27695-7609
}

\section{Raymond L. Clark ${ }^{3}$ \\ U.S. Department of Agriculture Regional Plant Introduction Station, Ames, IA 50011 \\ Additional index words. Cucumis sativus, fruiting, germplasm evaluation, vegetable breeding, fruit number}

\begin{abstract}
Cucumber (Cucumis sativus L.) plant introduction (PI) accessions from the regional PI station at Ames, Iowa were evaluated in open-field production for singleharvest yield at Clinton, N.C. and Ames, Iowa. Check cultivars and experimental inbreds were also tested for comparison with the PI accessions (the three groups hereafter collectively referred to as cultigens). In order to make the evaluation more uniform for all cultigens regardless of sex expression and fruit size, all were crossed with Gy 14, a gynoecious pickling cucumber inbred used commonly in the production of commercial hybrids. The resulting $\mathbf{7 6 1}$ gynoecious hybrids were tested for early, total, and marketable yield using recommended cultural practices. Results were obtained for 725 cultigens at both locations. Significant differences were observed among cultigens for all traits evaluated. Differences between the two locations were also significant for total yield, corrected total yield, and percentage of early fruit. The interaction of cultigen and location was significant for standardized total yield and standardized corrected total yield. The highest yielding hybrids at both locations were produced using the following cultigens as male (paternal) parents: PI 422185, PI 390253, PI 175120, PI 173889, PI 267087, PI 175686, PI 178888, PI 385967, PI 458851, and PI 171601. The highest and lowest yielding paternal parents from the germplasm screening study were retested, along with check cultigens in a multiple-harvest trial at Clinton, N.C. Cultigens were evaluated directly, rather than as hybrids with $\mathrm{Gy} \mathrm{14}$, and fruit number, fruit weight, and sex expression were recorded. Most cultigens performed as expected for the yield traits in the retest study. The exceptions were 'Wautoma' and PI 339250, which were among the low and high yielders in the first test, but were ranked as medium and low, respectively, in the retest study.
\end{abstract}

Cucumber is thought to have originated in India (Harlan, 1975), with domestication occurring later throughout Europe. Breeding for yield has been one of the important objectives of many cucumber breeding programs since the 1900s (Wehner, 1989). Yield of pickling cucumber has been improved by incorporating disease resistance into culti-

\footnotetext{
Received for publication 7 Sept. 1999. Accepted for publication $14 \mathrm{Feb} .2000$. The use of trade names in this publication does not imply endorsement by the North Carolina Agricultural Research Service (NCARS) of the products named, nor criticism of similar ones not mentioned. Research funded in part by a grant from the North Carolina Pickle Producers Association. We gratefully acknowledge the technical assistance of R.R. Horton, Jr. The cost of publishing this paper was defrayed in part by the payment of page charges. Under postal regulations, this paper therefore must be hereby marked advertisement solely to indicate this fact.

${ }^{1}$ To whom requests for reprints should be addressed. E-mail address: todd_wehner@ncsu.edu

${ }^{2}$ Professor and Graduate Research Assistant, respectively.

${ }^{3}$ Research Leader (retired).
}

vars (Peterson, 1975) and using improved cultural practices (Cargill et al., 1975). Increased yield has also resulted from improvement of qualitative traits such as gynoecious sex expression and uniform green fruit color (improved percentage of marketable fruit) (Wehner, 1989).

However, improvement in yield in recent years has been limited. Additional yield improvement might be achieved by identifying new sources of germplasm for high yield. The cucumber germplasm collection is a good place to find new sources of high yielding cultigens because no previous work has been done on screening the collection for yield. However, measurement of yield in a diverse array of cucumber cultigens can be difficult. Yield is usually measured as fruit number, weight, or value per unit area or per plant. Fruit number was more stable than fruit weight or fruit value for yield measurement in a once-over harvest trial of cucumber (Ells and McSay, 1981) and had a higher heritability $(0.17)$ than fruit weight (0.02) (Smith and Lower, 1978).

Large-plot, multiple-harvest trials in multiple years, locations, seasons, and repli- cations would be ideal for yield measurement. However, it would be nearly impossible to include $>700$ cultigens in such trials. Single harvest trials are efficient for yield measurement, but introduce the problem of the optimum time to harvest each plot for maximum yield. Miller and Hughes (1969) reported that harvesting when $14 \%$ to $31 \%$ of the fruits in a plot were oversized $(>51 \mathrm{~mm}$ diameter for pickling cucumber and $>60 \mathrm{~mm}$ diameter for fresh market cucumber) was optimum for maximum value in once-over harvest for 'Piccadilly' and 'Southern Cross' gynoecious hybrids in North Carolina. Chen et al. (1975), using a computer simulation, reported that plots harvested at $10 \%$ oversized fruit stage gave an optimum yield for 'Piccadilly' hybrid under North Carolina conditions. Colwell and O'Sullivan (1981) reported that the optimum harvest stage to maximize yield for 'Femcap' and 'Greenstar' gynoecious hybrids occurred when $5 \%$ to $15 \%$ of the fruit in a plot were oversized.

Small-plot trials are efficient, but plots should not be too small. Yield of cultivars in single-plant hills was poorly correlated with large, replicated plots harvested several times (Wehner, 1986; Wehner and Miller, 1984). In addition, greenhouse evaluation of yield, based on fruit number of single plants, was not correlated $(r=0.09-0.15)$ with yield in field trials (Nerson et al., 1987). Once-over harvest trials with three replications are optimum for determining which cucumber cultigens should be tested further in multiple-harvest trials (Wehner, 1986; Wehner and Miller, 1984). A plot size of $1.2 \mathrm{~m} \times 1.5 \mathrm{~m}$ was found to be optimum for yield evaluation of pickling cucumber when paraquat (1,1'-dimethyl-4,4'bipyridinium ion) was used to simulate onceover harvest (Swallow and Wehner, 1986). In cucumber, small-plot, single-harvest trials were more efficient than large-plot, multiple-harvest trials (Wehner, 1986, 1989; Wehner and Miller, 1987).

Swallow and Wehner (1989) suggested that maximum information (1/variance) was obtained by allocating test plots of cucumber cultigens over different seasons and years rather than different locations and replications. However, use of locations and replications was less expensive than use of seasons and years. Field evaluation at the Clinton location was more efficient than at three other locations tested in North Carolina (Wehner, 1987a).

The $>700$ cultigens in the U.S. Dept. of Agriculture(USDA) cucumber germplasm collection range from androecious to monoecious to gynoecious. Thus, measuring yield of a diverse set of cultigens in a single-harvest trial is difficult, even though that method is the most efficient. Some of the cultigens are highly staminate in flowering habit, so will not produce much fruit. Even so, androecious cultigens might have useful genes for yield. For that reason, we crossed each cultigen with Gy 14 to make gynoecious hybrids. In that way, yield of all cultigens from the germplasm collection could be expressed in a gynoecious background with the potential to set fruit at many nodes. By measuring the combining 
Table 1. Fruit yield traits (yield in 1000 fruit/ha) ${ }^{2}$ for the cucumber germplasm collection tested at two locations for combining ability with Gy 14 (cultigens ranked by standardized corrected total fruit number over both locations).

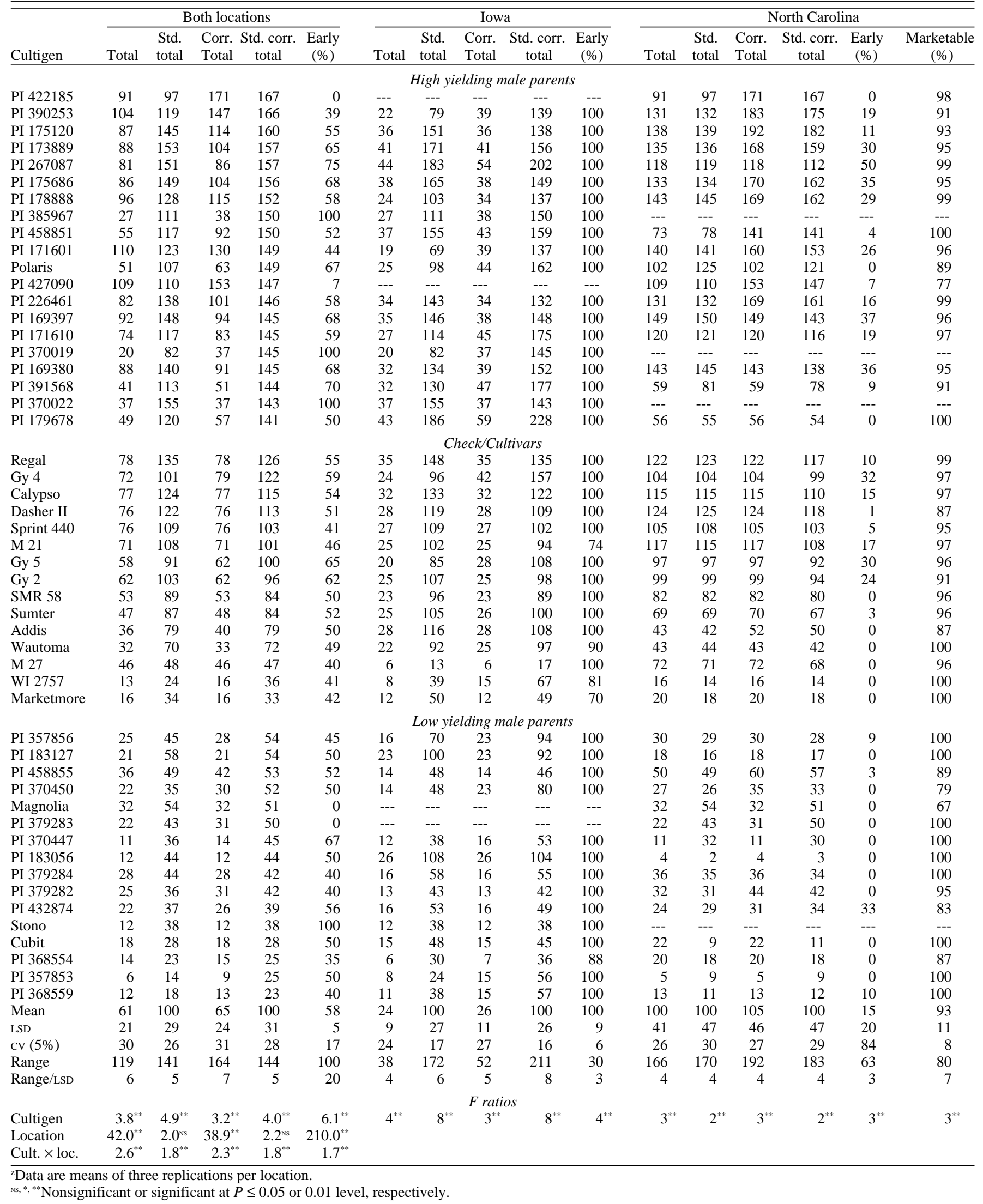


ability of yield, we would be able to identify cultigens that contributed useful, dominant genes for yield to their hybrid.

A disadvantage of measuring yield of the germplasm collection as combining ability with a gynoecious tester is that cultigens with recessive genes for yield would not perform as well. Therefore, cultigens that rank high in this evaluation of combining ability for yield would be excellent choices for use in a breeding program for yield, but cultigens that do not rank high might also be excellent choices. Therefore, low performers should be retested for yield using methods other than combining ability.
In the breeding program at North Carolina State Univ., we intend to measure yield of the cucumber germplasm collection using a threestage process. First, testing of all available cultigens using combining ability with a gynoecious tester in small-plot, single-harvest trials. Second, testing of all available cultigens for yield per se in small-plot, single-harvest trials after treating the plants with ethrel (2-chloroethylphosphonic acid) to increase gynoecious sex expression. Third, evaluation of the high-yielding cultigens from the previous two stages using large-plot, multiple-harvest trials in multiple seasons and years. This study deals with the first stage of that process.
The objective of this study was to evaluate all available cucumber cultigens for combining ability for yield at two locations using a gynoecious tester. Identification of the highest yielders was followed by a retest of performance per se to verify performance for use in yield improvement in breeding programs for commercial cultivars.

\section{Materials and Methods}

All experiments were conducted at the Horticultural Crops Research Station, Clinton, N.C., and the Regional Plant Introduction Station, Ames, Iowa. The experiments were

Table 2. Correlations among fruit yield traits for the cucumber germplasm collection tested at two locations.

\begin{tabular}{|c|c|c|c|c|c|c|c|c|c|c|c|c|c|c|c|}
\hline \multirow[b]{2}{*}{ Cultigen } & \multicolumn{4}{|c|}{ Both locations } & \multicolumn{5}{|c|}{ Iowa } & \multicolumn{6}{|c|}{ North Carolina } \\
\hline & $\begin{array}{l}\text { Std. } \\
\text { total }\end{array}$ & $\begin{array}{l}\text { Corr. } \\
\text { total }\end{array}$ & $\begin{array}{c}\text { Std. Corr. } \\
\text { total }\end{array}$ & $\begin{array}{c}\text { Early } \\
(\%)\end{array}$ & Total & $\begin{array}{c}\text { Std. } \\
\text { total }\end{array}$ & $\begin{array}{l}\text { Corr. } \\
\text { total }\end{array}$ & $\begin{array}{c}\text { Std. Corr. } \\
\text { total }\end{array}$ & $\begin{array}{c}\text { Early } \\
(\%)\end{array}$ & Total & $\begin{array}{l}\text { Std. } \\
\text { total }\end{array}$ & $\begin{array}{l}\text { Corr. } \\
\text { total }\end{array}$ & $\begin{array}{c}\text { Std. Corr. } \\
\text { total }\end{array}$ & $\begin{array}{c}\text { Early } \\
(\%)\end{array}$ & $\begin{array}{c}\text { Marketable } \\
(\%)\end{array}$ \\
\hline \multicolumn{16}{|c|}{ Both locations } \\
\hline Total & 0.81 & 0.92 & 0.73 & -0.14 & 0.44 & 0.43 & 0.30 & 0.31 & 0.13 & 0.92 & 0.91 & 0.87 & 0.86 & 0.44 & 0.04 \\
\hline Standardized total & --- & 0.69 & 0.86 & 0.26 & 0.76 & 0.77 & 0.54 & 0.56 & 0.16 & 0.85 & 0.86 & 0.78 & 0.78 & 0.47 & 0.12 \\
\hline Corrected total & & --- & 0.79 & -0.27 & 0.40 & 0.39 & 0.39 & 0.39 & 0.14 & 0.77 & 0.77 & 0.89 & 0.89 & 0.37 & 0.00 \\
\hline Standardized corre & otal & & --- & 0.15 & 0.67 & 0.66 & 0.72 & 0.74 & 0.17 & 0.73 & 0.75 & 0.81 & 0.83 & 0.37 & 0.06 \\
\hline Early yield (\%) & & & & --- & 0.17 & 0.19 & 0.05 & 0.08 & 0.10 & 0.26 & 0.26 & 0.16 & 0.15 & 0.63 & 0.15 \\
\hline \multicolumn{16}{|c|}{ Iowa } \\
\hline Total & & & & & --- & 0.99 & 0.76 & 0.76 & 0.17 & 0.33 & 0.33 & 0.32 & 0.32 & 0.25 & 0.14 \\
\hline Standardized total & & & & & & --- & 0.73 & 0.75 & 0.16 & 0.34 & 0.34 & 0.32 & 0.32 & 0.26 & 0.15 \\
\hline Corrected total & & & & & & & --- & 0.99 & 0.16 & 0.18 & 0.19 & 0.21 & 0.22 & 0.14 & 0.08 \\
\hline Standardized corre & otal & & & & & & & --- & 0.15 & 0.19 & 0.21 & 0.21 & 0.23 & 0.16 & 0.10 \\
\hline Early yield (\%) & & & & & & & & & --- & 0.10 & 0.10 & 0.12 & 0.12 & 0.08 & 0.04 \\
\hline \multicolumn{16}{|c|}{ North Carolina } \\
\hline Total & & & & & & & & & & --- & 0.99 & 0.91 & 0.90 & 0.51 & 0.05 \\
\hline Standardized total & & & & & & & & & & & --- & 0.90 & 0.91 & 0.49 & 0.05 \\
\hline Corrected total & & & & & & & & & & & & --- & 0.42 & 0.02 & 0.02 \\
\hline Standardized corre & otal & & & & & & & & & & & & --- & 0.16 & 0.16 \\
\hline Early yield (\%) & & & & & & & & & & & & & & --- & 0.16 \\
\hline
\end{tabular}

Table 3. Mean sandardized corrected yield (1000 fruit/ha) for 761 cucumber cultigens tested at two locations (cultigens ranked by average yield at both locations.

\begin{tabular}{lccc}
\hline & & & \\
& & Fruit yield $^{\mathrm{y}}$ \\
\cline { 2 - 4 } Cultigen name & Seed source & North Carolina $^{\mathrm{z}}$ & Iowa $^{\mathrm{z}}$ \\
\hline PI 422185 & Czechoslovakia & 167 & --- \\
PI 390253 & Japan & 175 & 139 \\
PI 175120 & India & 182 & 138 \\
PI 173889 & India & 159 & 156 \\
PI 267087 & USSR & 112 & 202 \\
PI 175686 & Turkey & 162 & 149 \\
PI 178888 & Turkey & 162 & 137 \\
PI 385967 & Kenya & --- & 150 \\
PI 458851 & USSR & 141 & 159 \\
PI 171601 & Turkey & 153 & 137 \\
Polaris & NSSL & 121 & 162 \\
PI 427090 & P.R. China & 147 & --- \\
PI 226461 & Iran & 161 & 132 \\
PI 169397 & Turkey & 143 & 148 \\
PI 171610 & Turkey & 116 & 175 \\
PI 370019 & India & --- & 145 \\
PI 169380 & Turkey & 138 & 152 \\
PI 391568 & P.R. China & 78 & 177 \\
PI 370022 & India & --- & 143 \\
PI 179678 & India & 54 & 228 \\
PI 401732 & Puerto Rico & 140 & 140 \\
PI 344445 & Iran & 139 & 140 \\
PI 169391 & Turkey & 152 & 118 \\
PI 174172 & Turkey & 150 & 128 \\
PI 342950 & Denmark & 138 & 133 \\
PI 280096 & USSR & & 139 \\
& & &
\end{tabular}

Table 3. Continued.

\begin{tabular}{|c|c|c|c|}
\hline \multirow[b]{2}{*}{ Cultigen name } & \multirow[b]{2}{*}{ Seed source ${ }^{z}$} & \multicolumn{2}{|c|}{ Fruit yield $^{\mathrm{y}}$} \\
\hline & & North Carolina $^{z}$ & Iowa $^{z}$ \\
\hline PI 344442 & Iran & 173 & 86 \\
\hline PI 172846 & Turkey & 149 & 127 \\
\hline PI 205995 & Sweden & 132 & 146 \\
\hline PI 263083 & P.R. China & 122 & 151 \\
\hline PI 390953 & USSR & 88 & 152 \\
\hline Ansansky & NSSL & 136 & --- \\
\hline Producer & NSSL & 144 & 127 \\
\hline PI 354952 & Denmark & 108 & 162 \\
\hline PI 418963 & P.R. China & 141 & 127 \\
\hline PI 308916 & USSR & 116 & 151 \\
\hline PI 288992 & Hungary & 130 & 137 \\
\hline PI 357855 & Yugoslavia & 103 & 163 \\
\hline PI 167134 & Turkey & 147 & 118 \\
\hline PI 175690 & Turkey & 125 & 140 \\
\hline PI 222244 & Iran & 115 & 149 \\
\hline PI 175696 & Turkey & 132 & 132 \\
\hline PI 113334 & P.R. China & 130 & 134 \\
\hline Shamrock Resistant & NSSL & 173 & 91 \\
\hline PI 183231 & Egypt & 151 & 113 \\
\hline Pixie & NSSL & 130 & 133 \\
\hline PI 263047 & USSR & 131 & 132 \\
\hline PI 169393 & Turkey & 127 & 136 \\
\hline PI 419108 & P.R. China & 134 & 128 \\
\hline PI 169378 & Turkey & 135 & 127 \\
\hline PI 285605 & Poland & 133 & 128 \\
\hline PI 226510 & Iran & 125 & 135 \\
\hline PI 436648 & P.R. China & 112 & 147 \\
\hline PI 279465 & Japan & 121 & 155 \\
\hline
\end{tabular}


Breeding, Cultivars, Rootstocks, \& Germplasm Resources

Table 3. Continued.

\begin{tabular}{|c|c|c|c|}
\hline \multirow[b]{2}{*}{ Cultigen name } & \multirow[b]{2}{*}{ Seed source ${ }^{z}$} & \multicolumn{2}{|c|}{ Fruit yield $^{y}$} \\
\hline & & North Carolina $^{\mathrm{z}}$ & Iowa $^{z}$ \\
\hline PI 137844 & Iran & 146 & 112 \\
\hline PI 105340 & P.R. China & 129 & 129 \\
\hline PI 432873 & P.R. China & 99 & 174 \\
\hline PI 478365 & P.R. China & 123 & 138 \\
\hline PI 175693 & Turkey & 127 & 130 \\
\hline PI 118279 & Brazil & 136 & 121 \\
\hline PI 181753 & Syria & 141 & 116 \\
\hline PI 222243 & Iran & 127 & 130 \\
\hline PI 360939 & Netherlands & 105 & 163 \\
\hline PI 419010 & P.R. China & 134 & 120 \\
\hline PI 179676 & India & 88 & 168 \\
\hline PI 164950 & Turkey & 130 & 125 \\
\hline PI 171607 & Turkey & 132 & 123 \\
\hline PI 466922 & USSR & 118 & 141 \\
\hline PI 206425 & Turkey & 138 & 117 \\
\hline PI 339250 & Turkey & 151 & 103 \\
\hline PI 169304 & Turkey & 159 & 95 \\
\hline Long of Keschmet & NSSL & 100 & 136 \\
\hline PI 422176 & Czechoslovakia & 122 & 133 \\
\hline PI 288237 & Egypt & 125 & 128 \\
\hline PI 114339 & Japan & 118 & 134 \\
\hline PI 279468 & Japan & 96 & 156 \\
\hline PI 165499 & India & 117 & 135 \\
\hline PI 271328 & India & 118 & 134 \\
\hline PI 271326 & India & 104 & 148 \\
\hline Snows Pickling & NSSL & 111 & 141 \\
\hline PI 164951 & Turkey & 123 & 128 \\
\hline PI 422191 & Czechoslovakia & 148 & 92 \\
\hline Regal & Check $^{\mathrm{x}}$ & 117 & 135 \\
\hline PI 211988 & Iran & 111 & 140 \\
\hline PI 275410 & Netherlands & 118 & 133 \\
\hline PI 432875 & P.R. China & 126 & 124 \\
\hline PI 171613 & Turkey & 110 & 140 \\
\hline PI 390247 & Japan & 125 & --- \\
\hline PI 342951 & Denmark & 123 & 126 \\
\hline PI 172843 & Turkey & 148 & 101 \\
\hline PI 432889 & P.R. China & 149 & 87 \\
\hline PI 271754 & Netherlands & 127 & 121 \\
\hline PI 221440 & Afghanistan & 117 & 129 \\
\hline PI 432848 & P.R. China & 132 & 115 \\
\hline PI 326594 & Hungary & 128 & 119 \\
\hline PI 271753 & Netherlands & 119 & 128 \\
\hline PI 169398 & Turkey & 126 & 121 \\
\hline PI 177359 & Turkey & 122 & 125 \\
\hline PI 211982 & Iran & 118 & 127 \\
\hline PI 169392 & Turkey & 130 & 115 \\
\hline PI 175683 & Turkey & 141 & 104 \\
\hline PI 432850 & P.R. China & 123 & 122 \\
\hline Gy 4 & Check $^{x}$ & 99 & 157 \\
\hline PI 400270 & Japan & 145 & 87 \\
\hline PI 283900 & Czechoslovakia & 123 & 121 \\
\hline PI 204567 & Turkey & 122 & 122 \\
\hline PI 164819 & India & 123 & 120 \\
\hline PI 172841 & Turkey & 121 & 123 \\
\hline PI 422168 & Czechoslovakia & 117 & 128 \\
\hline PI 177364 & Iraq & 164 & 79 \\
\hline PI 302443 & P.R. China & 131 & 112 \\
\hline PI 204568 & Turkey & 119 & 124 \\
\hline PI 326597 & Hungary & 129 & 114 \\
\hline PI 174166 & Turkey & 118 & 124 \\
\hline PI 422179 & Czechoslovakia & 109 & 157 \\
\hline Tiny Dill & NSSL & 134 & 107 \\
\hline PI 249561 & Thailand & 110 & 131 \\
\hline PI 220860 & Korea & 110 & 131 \\
\hline PI 356809 & USSR & 130 & 111 \\
\hline PI 458852 & USSR & 136 & 98 \\
\hline PI 285609 & Poland & 121 & --- \\
\hline PI 137836 & Iran & 142 & 99 \\
\hline PI 174170 & Turkey & 125 & 115 \\
\hline PI 103049 & P.R. China & 124 & 117 \\
\hline PI 227207 & Japan & 115 & 125 \\
\hline PI 432894 & P.R. China & 129 & 111 \\
\hline PI 176951 & Turkey & 125 & 115 \\
\hline PI 262974 & India & 122 & 118 \\
\hline PI 165509 & India & 92 & 148 \\
\hline
\end{tabular}

Table 3. Continued.

\begin{tabular}{|c|c|c|c|}
\hline \multirow{2}{*}{ Cultigen name } & \multirow[b]{2}{*}{ Seed source ${ }^{\mathrm{z}}$} & \multicolumn{2}{|c|}{ Fruit yield $^{y}$} \\
\hline & & North Carolina ${ }^{z}$ & Iowa $^{2}$ \\
\hline PI 422173 & Czechoslovakia & 114 & 129 \\
\hline PI 264668 & Germany & 114 & 126 \\
\hline PI 193496 & Ethiopia & 128 & 111 \\
\hline PI 339247 & Turkey & 139 & 101 \\
\hline PI 196289 & India & 109 & 130 \\
\hline PI 355052 & Israel & 113 & 126 \\
\hline PI 478367 & P.R. China & 121 & 117 \\
\hline Texas Long & NSSL & 95 & 132 \\
\hline PI 267942 & Japan & 99 & 133 \\
\hline PI 169385 & Turkey & 134 & 105 \\
\hline PI 169403 & Turkey & 122 & 115 \\
\hline PSI & NSSL & 128 & 106 \\
\hline PI 263080 & USSR & 117 & 121 \\
\hline PI 181910 & Syria & 109 & 128 \\
\hline PI 478364 & P.R. China & 139 & 89 \\
\hline PI 175694 & Turkey & 137 & 101 \\
\hline PI 284699 & Sweden & 116 & 122 \\
\hline PI 109484 & Turkey & 111 & 126 \\
\hline PI 169328 & Turkey & 115 & 123 \\
\hline PI 422167 & Czechoslovakia & 124 & 116 \\
\hline PI 264230 & France & 121 & 116 \\
\hline PI 234517 & U.S.-S.C. & 117 & 119 \\
\hline PI 422184 & Czechoslovakia & 95 & 141 \\
\hline PI 314426 & USSR & 115 & 121 \\
\hline PI 188749 & Egypt & 126 & 109 \\
\hline PI 227664 & Iran & 96 & 132 \\
\hline PI 200815 & Burma & 114 & 129 \\
\hline PI 179921 & India & 116 & 119 \\
\hline PI 344349 & Turkey & 124 & 111 \\
\hline PI 181756 & Lebanon & 127 & 107 \\
\hline PI 357862 & Yugoslavia & 144 & 77 \\
\hline PI 172852 & Turkey & 112 & 122 \\
\hline PI 193497 & Ethiopia & 115 & 119 \\
\hline PI 422177 & Czechoslovakia & 109 & 125 \\
\hline PI 432892 & P.R. China & 120 & 114 \\
\hline Sunny South & NSSL & 131 & 95 \\
\hline PI 285607 & Poland & 124 & 109 \\
\hline PI 390252 & Japan & 105 & 151 \\
\hline PI 176517 & Turkey & 131 & 103 \\
\hline PI 182188 & Turkey & 119 & 115 \\
\hline PI 169395 & Turkey & 94 & 151 \\
\hline PI 351139 & USSR & 114 & 120 \\
\hline PI 174177 & Turkey & 121 & 112 \\
\hline PI 339241 & Turkey & 125 & 108 \\
\hline PI 181942 & Syria & 125 & 107 \\
\hline PI 269481 & Pakistan & 117 & 115 \\
\hline PI 105263 & Turkey & 107 & 125 \\
\hline PI 169401 & Turkey & 108 & 124 \\
\hline PI 357841 & Yugoslavia & 118 & 114 \\
\hline PI 451975 & Canada & 120 & 110 \\
\hline PI 137856 & Iran & 125 & 106 \\
\hline PI 487424 & P.R. China & 116 & --- \\
\hline PI 175695 & Turkey & 121 & 109 \\
\hline PI 267943 & Japan & 117 & 114 \\
\hline PI 176521 & Turkey & 116 & 115 \\
\hline Calypso & Check $^{\mathrm{x}}$ & 110 & 122 \\
\hline PI 174167 & Turkey & 116 & 114 \\
\hline PI 181940 & Syria & 113 & 122 \\
\hline PI 248778 & Iran & 136 & 93 \\
\hline PI 285603 & Poland & 122 & 108 \\
\hline PI 183677 & Turkey & 106 & 124 \\
\hline PI 137846 & Iran & 119 & 111 \\
\hline PI 137857 & Iran & 113 & 116 \\
\hline Early Green Cluster & NSSL & 114 & 115 \\
\hline PI 432865 & P.R. China & 130 & 91 \\
\hline PI 135345 & Afghanistan & 122 & 106 \\
\hline PI 264228 & France & 104 & 124 \\
\hline PI 422174 & Czechoslovakia & 77 & 170 \\
\hline PI 227209 & Japan & 103 & 131 \\
\hline PI 267741 & Japan & 108 & 124 \\
\hline PI 164816 & India & 108 & 120 \\
\hline PI 419079 & P.R. China & 110 & 118 \\
\hline PI 137848 & Iran & 107 & 121 \\
\hline PI 109482 & Turkey & 125 & 102 \\
\hline PI 092806 & P.R. China & & \\
\hline
\end{tabular}


conducted using recommended horticultural practices (Hughes et al., 1983; Schultheis, 1990). Fertilizer was incorporated before planting at a rate of $90 \mathrm{~N}-39 \mathrm{P}-74 \mathrm{~K} \mathrm{~kg} \cdot \mathrm{ha}^{-1}$, with an additional $34 \mathrm{~kg} \cdot \mathrm{ha}^{-1} \mathrm{~N}$ applied at the vine tip-over stage. Curbit ${ }^{\circledR}$ [(ethalfluralin $N$ ethyl- $N$-(2-methyl-2-propenyl)-2,6-dinitro-4(trifluoromethyl)benzenamine)] was applied for weed control. Irrigation was applied when needed for a total (irrigation plus rainfall) of 25 to $40 \mathrm{~mm}$ per week. 'Sumter' pollenizer was planted on the sides of the plots to provide pollen.

Plots were disease free through once-over harvest stage (26 June) when oversized fruit averaged $15 \%$ across all the plots in the field. Although the usual index for yield evaluation for testing populations in our breeding pro- gram is $10 \%$, we used a $15 \%$ index in this study to allow late-flowering cultigens to produce fruit.

Cultural practices. In North Carolina, seeds were planted on raised, shaped beds 1.5 $\mathrm{m}$ apart. Plots were $1.2 \mathrm{~m}$ long $\times 1.5 \mathrm{~m}$ wide with $1.2-\mathrm{m}$ alleys at each end. Guard rows were planted on the outside of the field, and at the end of each row. Plots were planted with 14 seeds on 12 May and thinned to a uniform stand of 10 plants per plot on 29 May.

In Iowa, seeds were planted in beds $1.5 \mathrm{~m}$ apart. Plots were $6.0 \mathrm{~m}$ long and $1.5 \mathrm{~m}$ wide with $1.5-\mathrm{m}$ alleys at each end. Guard rows were planted on the outside of the field, and at the end of each row using 'Sumter' as a pollenizer. The plots were planted with 25 seeds on 12 May and thinned to a uniform stand of 20 plants per plot.

Cultigens evaluated. In this experiment, 761 cultigens (746 PI accessions and 15 check cultivars and breeding lines) were evaluated. Accessions designated with a PI number were obtained from the USDA germplasm collection in Ames, Iowa. Cultigens with an NSSL seed source were obtained from the National Seed Storage Laboratory in Fort Collins, Colo. The cultigens originated from 44 different countries. Countries with the most cultigens were Turkey (166), the People's Republic of China (P.R. China) (85), Iran (64), the former Yugoslavia (63), India (47), Japan (44), the former Soviet Union (USSR) (37), the former Czechoslovakia (27), The Netherlands (19), United States (16), and
Table 3. Continued.

\begin{tabular}{|c|c|c|c|}
\hline \multirow[b]{2}{*}{ Cultigen name } & \multirow[b]{2}{*}{ Seed source ${ }^{z}$} & \multicolumn{2}{|c|}{ Fruit yieldy } \\
\hline & & North Carolina $^{z}$ & Iowa $^{z}$ \\
\hline PI 483344 & Korea & 35 & 192 \\
\hline PI 263046 & USSR & 105 & 122 \\
\hline Dasher II & Check $^{\mathrm{x}}$ & 118 & 109 \\
\hline PI 209064 & U.S.-Ohio & 104 & 123 \\
\hline PI 264667 & Germany & 141 & 85 \\
\hline PI 414157 & U.S.-Ore. & 96 & 125 \\
\hline PI 171602 & Turkey & 116 & 110 \\
\hline PI 288238 & Egypt & 114 & 113 \\
\hline PI 324239 & Sweden & 120 & 106 \\
\hline PI 344434 & Iran & 109 & 117 \\
\hline PI 458849 & USSR & 130 & 95 \\
\hline PI 285606 & Poland & 120 & 106 \\
\hline PI 169384 & Turkey & 113 & --- \\
\hline PI 172845 & Turkey & 127 & 98 \\
\hline PI 436672 & P.R. China & 106 & 122 \\
\hline PI 212233 & Japan & 110 & 115 \\
\hline PI 181874 & Syria & 105 & 120 \\
\hline PI 356832 & Netherlands & 108 & 116 \\
\hline PI 176957 & Turkey & 109 & 115 \\
\hline PI 109481 & Turkey & 113 & 112 \\
\hline PI 172849 & Turkey & 108 & 116 \\
\hline PI 289698 & Australia & 114 & 111 \\
\hline PI 422171 & Czechoslovakia & 96 & 136 \\
\hline PI 169396 & Turkey & 100 & 124 \\
\hline PI 435946 & USSR & 112 & 113 \\
\hline PI 171604 & Turkey & 112 & 113 \\
\hline PI 167389 & Turkey & 110 & 114 \\
\hline PI 174174 & Turkey & 115 & 109 \\
\hline PI 263048 & USSR & 106 & 117 \\
\hline PI 255938 & Netherlands & 131 & 92 \\
\hline PI 209068 & U.S.-Ohio & 110 & 113 \\
\hline PI 379278 & Yugoslavia & 103 & 125 \\
\hline PI 264665 & Germany & 109 & 114 \\
\hline PI 267746 & India & 116 & 107 \\
\hline PI 172847 & Turkey & 117 & 106 \\
\hline Clinton & N.C. State Univ. & 110 & 114 \\
\hline PI 392292 & USSR & 103 & 125 \\
\hline PI 176525 & Turkey & 137 & 86 \\
\hline PI 422172 & Czechoslovakia & 97 & 154 \\
\hline PI 255933 & Netherlands & 122 & 100 \\
\hline PI 172848 & Turkey & 116 & 105 \\
\hline PI 211977 & Iran & 110 & 112 \\
\hline PI 171612 & Turkey & 116 & 106 \\
\hline PI 211962 & Iran & 94 & 127 \\
\hline PI 344351 & Turkey & 106 & 115 \\
\hline Boston Pickling & NSSL & 94 & 136 \\
\hline PI 419183 & P.R. China & 138 & 92 \\
\hline PI 263082 & P.R. China & 113 & 108 \\
\hline PI 390258 & Japan & 78 & 143 \\
\hline PI 308915 & USSR & 110 & 111 \\
\hline Robin & NSSL & 46 & 142 \\
\hline PI 178886 & Turkey & 113 & 107 \\
\hline
\end{tabular}

Table 3. Continued.

\begin{tabular}{|c|c|c|c|}
\hline \multirow[b]{2}{*}{ Cultigen name } & \multirow[b]{2}{*}{ Seed source ${ }^{z}$} & \multicolumn{2}{|c|}{ Fruit yield $^{y}$} \\
\hline & & North Carolina ${ }^{z}$ & Iowa $^{z}$ \\
\hline PI 279464 & Japan & 115 & 105 \\
\hline PI 224668 & Korea & 80 & 141 \\
\hline PI 432883 & P.R. China & 113 & 107 \\
\hline PI 172844 & Turkey & 108 & 112 \\
\hline PI 344353 & Turkey & 112 & 108 \\
\hline PI 390259 & Japan & 83 & 138 \\
\hline PI 343452 & USSR & 122 & 98 \\
\hline PI 169394 & Turkey & 114 & 106 \\
\hline PI 271331 & India & 103 & 116 \\
\hline PI 137853 & Iran & 120 & 99 \\
\hline PI 279463 & Japan & 107 & 113 \\
\hline PI 483342 & Korea & 105 & 116 \\
\hline PI 182190 & Turkey & 108 & 111 \\
\hline PI 436609 & P.R. China & 113 & 107 \\
\hline PI 418962 & P.R. China & 108 & 111 \\
\hline PI 391570 & P.R. China & --- & 109 \\
\hline PI 339246 & Turkey & 126 & 92 \\
\hline PI 264664 & Germany & 95 & 124 \\
\hline PI 175689 & Turkey & 117 & 101 \\
\hline PI 344350 & Turkey & 100 & 118 \\
\hline PI 321006 & Taiwan & 113 & 104 \\
\hline PI 390269 & Japan & 97 & 121 \\
\hline PI 355053 & Iran & 101 & 117 \\
\hline PI 211978 & Iran & 132 & 85 \\
\hline Staygreen & NSSL & 101 & 120 \\
\hline PI 419136 & P.R. China & 122 & 96 \\
\hline PI 368548 & Yugoslavia & 84 & 145 \\
\hline PI 419017 & P.R. China & 123 & 87 \\
\hline PI 182192 & Turkey & 110 & 107 \\
\hline PI 137835 & Iran & 117 & 100 \\
\hline PI 169388 & Turkey & 106 & 112 \\
\hline PI 369717 & Poland & 111 & 104 \\
\hline PI 173892 & India & 95 & 122 \\
\hline PI 357850 & Yugoslavia & 115 & 102 \\
\hline Giant White Arnstadt & lt NSSL & --- & 108 \\
\hline PI 106063 & P.R. China & 107 & 110 \\
\hline PI 390255 & Japan & 90 & 127 \\
\hline PI 257494 & Iran & 112 & 105 \\
\hline PI 283899 & Czechoslovakia & 104 & 112 \\
\hline PI 209065 & U.S.-Ohio & 107 & 108 \\
\hline PI 175680 & Turkey & 107 & 108 \\
\hline PI 263084 & P.R. China & 126 & 90 \\
\hline PR 39 & NSSL & 69 & 146 \\
\hline PI 257286 & Spain & 106 & 109 \\
\hline PI 432864 & P.R. China & 80 & 150 \\
\hline PI 357851 & Yugoslavia & 98 & 117 \\
\hline PI 211117 & Israel & 113 & 102 \\
\hline PI 211980 & Iran & 107 & 108 \\
\hline PI 209069 & U.S.-Ohio & 95 & 119 \\
\hline PI 357858 & Yugoslavia & 113 & 99 \\
\hline PI 288990 & Hungary & 90 & 124 \\
\hline PI 288996 & Hungary & 116 & 98 \\
\hline
\end{tabular}


Breeding, Cultivars, Rootstocks, \& Germplasm Resources

Table 3. Continued.

\begin{tabular}{|c|c|c|c|}
\hline \multirow[b]{2}{*}{ Cultigen name } & \multirow[b]{2}{*}{ Seed source ${ }^{z}$} & \multicolumn{2}{|c|}{ Fruit yield ${ }^{y}$} \\
\hline & & North Carolina ${ }^{z}$ & Iowa $^{2}$ \\
\hline PI 288995 & Hungary & 95 & 120 \\
\hline PI 376063 & Israel & 119 & 89 \\
\hline PI 171605 & Turkey & 77 & 137 \\
\hline PI 401734 & Puerto Rico & 123 & 91 \\
\hline PI 288993 & Hungary & 108 & 106 \\
\hline PI 172838 & Turkey & 122 & 92 \\
\hline PI 432853 & P.R. China & 108 & 105 \\
\hline PI 169319 & Turkey & 112 & 101 \\
\hline PI 169350 & Turkey & 110 & 103 \\
\hline PI 175679 & Turkey & 117 & 96 \\
\hline PI 172842 & Turkey & 105 & 108 \\
\hline PI 267747 & U.S.-Okla. & 118 & 95 \\
\hline PI 167197 & Turkey & 134 & 79 \\
\hline PI 419214 & Hong Kong & 121 & 91 \\
\hline PI 183224 & Egypt & 125 & 88 \\
\hline PI 212985 & India & 107 & 105 \\
\hline PI 246930 & Afghanistan & 110 & 103 \\
\hline PI 435947 & USSR & 89 & 132 \\
\hline PI 267743 & P.R. China & 98 & 115 \\
\hline PI 167050 & Turkey & 99 & 111 \\
\hline PI 164670 & India & 83 & 129 \\
\hline PR 27 & NSSL & 92 & 120 \\
\hline PI 264226 & France & 86 & 126 \\
\hline PI 422198 & Czechoslovakia & 78 & 134 \\
\hline PI 321007 & Taiwan & 103 & 109 \\
\hline PI 281448 & Korea & 99 & 113 \\
\hline PI 489754 & P.R. China & 124 & 78 \\
\hline PI 357842 & Yugoslavia & 100 & 111 \\
\hline PI 165046 & Turkey & 114 & 100 \\
\hline Chicago Pickling & NSSL & 118 & 82 \\
\hline PI 338235 & Turkey & 98 & 113 \\
\hline PI 390267 & Japan & 114 & 93 \\
\hline Nappa 63 & NSSL & 92 & 119 \\
\hline PI 357852 & Yugoslavia & 101 & 110 \\
\hline PI 211983 & Iran & 101 & 110 \\
\hline PI 267745 & Brazil & 88 & 122 \\
\hline PI 275412 & Netherlands & 111 & 100 \\
\hline PI 344435 & Iran & 113 & 93 \\
\hline PI 368550 & Yugoslavia & 78 & 133 \\
\hline PI 211985 & Iran & 115 & 95 \\
\hline PI 169386 & Turkey & 110 & 101 \\
\hline PI 206043 & Puerto Rico & 97 & 113 \\
\hline PI 357838 & Yugoslavia & 73 & 137 \\
\hline PI 164952 & Turkey & 122 & 88 \\
\hline PI 251520 & Iran & 100 & 110 \\
\hline PI 292010 & Israel & 103 & 107 \\
\hline PI 432877 & P.R. China & 93 & 129 \\
\hline PI 206954 & Turkey & 105 & --- \\
\hline PI 306179 & Poland & 98 & 112 \\
\hline Earliest of All & NSSL & 93 & 116 \\
\hline PI 418964 & P.R. China & 108 & 100 \\
\hline PI 422170 & Czechoslovakia & 92 & 117 \\
\hline PI 257486 & P.R. China & 103 & 106 \\
\hline PI 169377 & Turkey & 80 & 129 \\
\hline PI 179260 & Turkey & 110 & 99 \\
\hline PI 262990 & Netherlands & 110 & 99 \\
\hline PI 372898 & Netherlands & 83 & 147 \\
\hline PI 267197 & P.R. China & 115 & 94 \\
\hline PI 432870 & P.R. China & 134 & 74 \\
\hline PI 344067 & Turkey & 126 & 82 \\
\hline PI 419009 & P.R. China & 104 & 105 \\
\hline PI 171609 & Turkey & 89 & 119 \\
\hline PI 249550 & Iran & 99 & 109 \\
\hline Favor II & NSSL & 98 & 110 \\
\hline PI 177363 & Syria & 112 & 96 \\
\hline PI 226509 & Iran & 105 & 103 \\
\hline PI 432878 & P.R. China & --- & 104 \\
\hline PI 169383 & Turkey & 120 & 87 \\
\hline PI 169382 & Turkey & 102 & 105 \\
\hline PI 169389 & Turkey & 112 & 95 \\
\hline PI 197086 & India & 99 & 108 \\
\hline PI 229309 & Iran & 108 & 99 \\
\hline PI 171606 & Turkey & 109 & 98 \\
\hline PI 176522 & Turkey & 100 & 105 \\
\hline PI 339245 & Turkey & 104 & 102 \\
\hline
\end{tabular}

Table 3. Continued.

\begin{tabular}{|c|c|c|c|}
\hline \multirow[b]{2}{*}{ Cultigen name } & \multirow[b]{2}{*}{ Seed source ${ }^{z}$} & \multicolumn{2}{|c|}{ Fruit yield $^{y}$} \\
\hline & & North Carolina ${ }^{\mathrm{z}}$ & Iowa $^{2}$ \\
\hline PI 321010 & Taiwan & 87 & 119 \\
\hline PI 227208 & Japan & 104 & 102 \\
\hline PI 357839 & Yugoslavia & 118 & 88 \\
\hline PI 172851 & Turkey & 96 & 110 \\
\hline PI 269480 & Pakistan & 115 & 92 \\
\hline PI 343451 & USSR & 112 & 94 \\
\hline Arlington Wt. Spine & NSSL & 117 & 89 \\
\hline PI 174164 & Turkey & 109 & 99 \\
\hline PI 274902 & Great Britain & 115 & 91 \\
\hline Sprint 440 & Check $^{x}$ & 103 & 102 \\
\hline PI 137847 & Iran & 103 & 102 \\
\hline PI 264229 & France & 99 & 106 \\
\hline PI 432861 & P.R. China & 111 & 97 \\
\hline PI 173674 & Turkey & 117 & 88 \\
\hline PI 217644 & India & 109 & 96 \\
\hline PI 293923 & U.S.-S.C. & 129 & 76 \\
\hline PI 164734 & India & 101 & 103 \\
\hline PI 169353 & Turkey & 110 & 94 \\
\hline PI 176519 & Turkey & 102 & --- \\
\hline PI 344444 & Iran & 118 & 86 \\
\hline PI 176952 & Turkey & 118 & 86 \\
\hline PI 344439 & Iran & 98 & 107 \\
\hline PI 204569 & Turkey & 122 & 83 \\
\hline PI 176516 & Turkey & 111 & 93 \\
\hline PI 183445 & India & 90 & 114 \\
\hline PI 171600 & Turkey & 111 & 93 \\
\hline PI 390256 & Japan & 116 & 81 \\
\hline PI 167198 & Turkey & 107 & 97 \\
\hline PI 432866 & P.R. China & 103 & 100 \\
\hline PI 169315 & Turkey & 103 & 100 \\
\hline PI 171611 & Turkey & 106 & 98 \\
\hline PI 293432 & Lebanon & 102 & --- \\
\hline PI 326595 & Hungary & 110 & 93 \\
\hline PI 285608 & Poland & 111 & 92 \\
\hline PI 172840 & Turkey & 86 & 117 \\
\hline PI 422190 & Czechoslovakia & 87 & 117 \\
\hline PI 228344 & Iran & 90 & 113 \\
\hline National Pickling & NSSL & 112 & 87 \\
\hline PI 489752 & P.R. China & 102 & 101 \\
\hline PI 182189 & Turkey & 108 & 95 \\
\hline PI 432886 & P.R. China & 91 & 108 \\
\hline PI 458847 & USSR & 101 & --- \\
\hline PI 357859 & Yugoslavia & 102 & 101 \\
\hline PI 220790 & Afghanistan & 117 & 85 \\
\hline M 21 & Check $^{\mathrm{x}}$ & 108 & 94 \\
\hline PI 304803 & U.S.-N.Y. & 89 & 113 \\
\hline PI 419041 & P.R. China & --- & 101 \\
\hline PI 192940 & P.R. China & 91 & 111 \\
\hline PI 211943 & Iran & 103 & 97 \\
\hline PI 482464 & Zimbabwe & 107 & 92 \\
\hline Model & NSSL & 105 & 95 \\
\hline PI 422188 & Czechoslovakia & 97 & 106 \\
\hline PI 179263 & Turkey & 111 & 90 \\
\hline PI 211975 & Iran & 87 & 114 \\
\hline PI 233932 & Canada & 101 & 99 \\
\hline PI 137845 & Iran & 106 & 94 \\
\hline PI 169402 & Turkey & 112 & 88 \\
\hline PI 339244 & Turkey & 74 & 126 \\
\hline PI 163221 & India & 88 & 113 \\
\hline PI 176953 & Turkey & 75 & 125 \\
\hline PI 368556 & Yugoslavia & 92 & 112 \\
\hline PI 344348 & Turkey & 103 & 97 \\
\hline PI 326598 & Hungary & 90 & 109 \\
\hline Gy 5 & Check $^{\mathrm{x}}$ & 92 & 108 \\
\hline PI 356833 & Great Britain & 122 & 78 \\
\hline Palmetto & NSSL & 117 & 83 \\
\hline Straight 8 & NSSL & 89 & 110 \\
\hline PI 227210 & Japan & 100 & --- \\
\hline PI 391573 & P.R. China & 100 & 99 \\
\hline PI 227013 & Iran & 86 & 113 \\
\hline PI 222782 & Iran & 111 & 87 \\
\hline PI 167079 & Turkey & 108 & 90 \\
\hline PI 261609 & Spain & 98 & 100 \\
\hline PI 263079 & USSR & 96 & 102 \\
\hline PI 267086 & USSR & 67 & 131 \\
\hline
\end{tabular}


Afghanistan (14). The cultigens designated as checks were tested as the cultigens per se (not their $F_{1}$ with Gy 14).

The 761 cultigens consisted of PI accessions, obsolete cultivars, current cultivars, and experimental inbreds and hybrids. In order to produce gynoecious hybrids, the cultigens were crossed with Gy 14, a popular, publicly-released, gynoecious pickling cucumber inbred. All crosses were made by hand at the Horticultural Science greenhouses, Raleigh, during the previous year. The hybrids could then be evaluated for yield regardless of sex expression of the male parent. In order to represent each cultigen properly, each hybrid was made up from a mixture of seeds obtained by crossing several plants of each accession with Gy 14 .

Traits measured. Data were collected as plot means, and consisted of number of plants per plot, and number of early, total, and cull fruit per plot in both locations. Early fruit were the number of oversized fruit at harvest $(>51$ $\mathrm{mm}$ diameter for pickles and $>60 \mathrm{~mm}$ for slicing cucumber). The number of marketable fruit was calculated as total fruit minus culls. Cull fruits were misshapen (crooked or nubbined). Data for marketable fruits were not recorded in Iowa.

Data analysis. The experiment was a randomized complete-block design with 761 cultigens and three replications at two locations. Data were analyzed using GLM and CORR procedures of SAS (SAS Institute, Cary, N.C.). Yield traits were expressed as thousands of fruit/ha for ease of comparison of yield over locations, and with previously published studies.

The data were analyzed using actual yield and corrected yield for both locations. Plots with a stand count (plant number) of $<50 \%$ of the expected 20 plants were eliminated from the statistical analysis. Plots with stand counts ranging from $50 \%$ to $75 \%$ were corrected according to Cramer and Wehner (1998), using the formula: corrected yield $=($ total yield $/$ stand count $) \times 20$. The corrected yield and total yield were then standardized to a mean of 100 and a SD of 30 (a SD of 30 would give a range of 0 to 200 if individuals were within three SD of the mean) for each location and replication using the STANDARD procedure of SAS. Standardization permitted comparison of cultigens when some plots were missing from locations or replications. The most useful traits are those where the range/LSD is large. If the range/LSD is small $(<1)$, then the best cultigen cannot be statistically separated from the worst cultigen in the test.
Table 3. Continued.

\begin{tabular}{|c|c|c|c|}
\hline \multirow[b]{2}{*}{ Cultigen name } & \multirow[b]{2}{*}{ Seed source ${ }^{2}$} & \multicolumn{2}{|c|}{ Fruit yield $^{y}$} \\
\hline & & North Carolina $^{2}$ & Iowa $^{2}$ \\
\hline PI 211979 & Iran & 87 & 111 \\
\hline PI 163214 & India & 94 & 103 \\
\hline PI 169352 & Turkey & 95 & 102 \\
\hline PI 169400 & Turkey & 89 & 108 \\
\hline PI 222987 & Iran & 94 & 103 \\
\hline PI 357847 & Yugoslavia & 94 & 103 \\
\hline PI 177360 & Turkey & 101 & 96 \\
\hline PI 390241 & Japan & 76 & 121 \\
\hline PI 169351 & Turkey & 93 & 104 \\
\hline PI 288994 & Hungary & 89 & 107 \\
\hline PI 458848 & USSR & 82 & 145 \\
\hline PI 379285 & Yugoslavia & 101 & 93 \\
\hline PI 222986 & Iran & 96 & 100 \\
\hline PI 220791 & Afghanistan & 87 & 114 \\
\hline PI 401733 & Puerto Rico & 76 & 105 \\
\hline PI 306785 & Canada & 112 & 83 \\
\hline PI 436610 & P.R. China & 116 & 88 \\
\hline PI 292012 & Israel & 92 & 106 \\
\hline PI 414158 & U.S.-Hawaii & 100 & 93 \\
\hline PI 458846 & USSR & 101 & 91 \\
\hline PI 172839 & Turkey & 95 & 99 \\
\hline PI 355055 & Iran & 99 & 95 \\
\hline PI 304805 & U.S.-N.Y. & 66 & 128 \\
\hline PI 368558 & Yugoslavia & 128 & 66 \\
\hline PI 339248 & Turkey & 103 & 91 \\
\hline PI 275411 & Netherlands & 120 & 73 \\
\hline PI 255936 & Netherlands & 100 & 94 \\
\hline PI 251519 & Iran & 90 & 103 \\
\hline PI 292011 & Israel & 97 & 96 \\
\hline PI 220171 & Afghanistan & 103 & 87 \\
\hline PI 167358 & Turkey & 110 & 83 \\
\hline PI 357863 & Yugoslavia & 103 & 87 \\
\hline PI 344441 & Iran & 106 & 87 \\
\hline PI 174173 & Turkey & 109 & 84 \\
\hline PI 277741 & Netherlands & 108 & 85 \\
\hline Gy 2 & Check $^{x}$ & 94 & 98 \\
\hline PI 390952 & USSR & 104 & 88 \\
\hline PI 176956 & Turkey & 94 & 98 \\
\hline PI 432860 & P.R. China & 99 & 92 \\
\hline PI 422186 & Czechoslovakia & 105 & 87 \\
\hline PI 222783 & Iran & 94 & 98 \\
\hline PI 137839 & Iran & 85 & 106 \\
\hline PI 263081 & P.R. China & 94 & 97 \\
\hline PI 135123 & New Zealand & 85 & 106 \\
\hline PI 200818 & Burma & 87 & 105 \\
\hline PI 344347 & Turkey & 110 & 81 \\
\hline PI 176524 & Turkey & 89 & 101 \\
\hline PI 414159 & U.S.-Hawaii & 79 & 111 \\
\hline PI 178884 & Turkey & 114 & 75 \\
\hline PI 165506 & India & 80 & 110 \\
\hline PI 174160 & Turkey & 96 & 94 \\
\hline
\end{tabular}

Table 3. Continued.

\begin{tabular}{|c|c|c|c|}
\hline \multirow[b]{2}{*}{ Cultigen name } & \multirow[b]{2}{*}{ Seed source ${ }^{z}$} & \multicolumn{2}{|c|}{ Fruit yield ${ }^{y}$} \\
\hline & & North Carolina ${ }^{z}$ & Iowa $^{2}$ \\
\hline PI 432862 & P.R. China & 92 & 99 \\
\hline PI 419135 & P.R. China & 110 & 72 \\
\hline PI 267744 & P.R. China & 91 & 98 \\
\hline PI 432857 & P.R. China & 86 & 103 \\
\hline PI 206955 & Turkey & 110 & 79 \\
\hline PI 296387 & Iran & 67 & 122 \\
\hline PI 267088 & USSR & 94 & 94 \\
\hline PI 223437 & Afghanistan & 89 & 99 \\
\hline PI 436673 & P.R. China & 110 & 79 \\
\hline Early Fortune & NSSL & 97 & 91 \\
\hline PI 326596 & Hungary & 96 & 92 \\
\hline PI 357844 & Yugoslavia & 105 & 83 \\
\hline PI 212599 & Afghanistan & 107 & 74 \\
\hline PI 390248 & Japan & 97 & 87 \\
\hline PI 373918 & England & 89 & 100 \\
\hline PI 357846 & Yugoslavia & --- & 94 \\
\hline PI 169387 & Turkey & 98 & 89 \\
\hline PI 390239 & Japan & 75 & 121 \\
\hline PI 167043 & India & 101 & 86 \\
\hline PI 432858 & P.R. China & 97 & 90 \\
\hline PI 264666 & Germany & 94 & 92 \\
\hline PI 169390 & Turkey & 83 & 103 \\
\hline PI 167052 & Turkey & 89 & 97 \\
\hline PI 264231 & France & 99 & 87 \\
\hline PI 164173 & India & 88 & 98 \\
\hline PI 344433 & Iran & 91 & 95 \\
\hline PI 390251 & Japan & 41 & 118 \\
\hline PI 357865 & Yugoslavia & 107 & 78 \\
\hline PI 422182 & Czechoslovakia & 101 & 84 \\
\hline PI 368551 & Yugoslavia & 96 & 87 \\
\hline PI 338234 & Turkey & 102 & 83 \\
\hline PI 211984 & Iran & 85 & 99 \\
\hline PI 344432 & Iran & 88 & 97 \\
\hline PI 211589 & Afghanistan & 104 & 81 \\
\hline PI 370448 & Yugoslavia & 97 & 84 \\
\hline York State Pickle & NSSL & 94 & 86 \\
\hline PI 175691 & Turkey & 104 & 84 \\
\hline PI 390250 & Japan & 82 & 108 \\
\hline PI 227235 & Iran & 85 & 99 \\
\hline PI 217946 & Pakistan & 91 & 93 \\
\hline PI 255935 & Netherlands & 113 & 71 \\
\hline PI 344443 & Iran & 109 & 74 \\
\hline PI 321011 & Taiwan & 96 & 87 \\
\hline PI 267935 & Japan & 100 & 84 \\
\hline PI 319216 & Un. Arab. Repub. & 101 & 82 \\
\hline PI 321009 & Taiwan & 94 & 88 \\
\hline PI 422181 & Czechoslovakia & 70 & 123 \\
\hline PI 165029 & Turkey & 93 & 90 \\
\hline PI 164284 & India & 84 & 98 \\
\hline PI 135122 & New Zealand & 81 & 101 \\
\hline PI 283902 & Czechoslovakia & 101 & 81 \\
\hline
\end{tabular}


Breeding, Cultivars, Rootstocks, \& Germplasm Resources

Table 3. Continued.

\begin{tabular}{|c|c|c|c|}
\hline \multirow[b]{2}{*}{ Cultigen name } & \multirow[b]{2}{*}{ Seed source ${ }^{z}$} & \multicolumn{2}{|c|}{ Fruit yield $^{y}$} \\
\hline & & North Carolina ${ }^{z}$ & Iowa $^{z}$ \\
\hline PI 222099 & Afghanistan & 94 & 88 \\
\hline PI 204692 & Turkey & 104 & 78 \\
\hline PI 179259 & Turkey & 115 & 67 \\
\hline PI 283901 & Czechoslovakia & 74 & 108 \\
\hline PI 279467 & Japan & 70 & 112 \\
\hline PI 164743 & India & 90 & 92 \\
\hline PI 169381 & Turkey & 78 & 104 \\
\hline PI 257487 & P.R. China & 84 & 100 \\
\hline PI 163217 & India & 97 & 84 \\
\hline PI 357868 & Yugoslavia & 78 & 102 \\
\hline PI 222985 & Iran & 84 & 94 \\
\hline PI 357867 & Yugoslavia & 82 & 98 \\
\hline PI 176954 & Turkey & 67 & 113 \\
\hline PI 220789 & Afghanistan & 97 & 83 \\
\hline PI 175681 & Turkey & 80 & 100 \\
\hline PI 432867 & P.R. China & 92 & 87 \\
\hline PI 285604 & Poland & 102 & 77 \\
\hline PI 390265 & Japan & 76 & 103 \\
\hline PI 357857 & Yugoslavia & 104 & 79 \\
\hline PI 458850 & USSR & 108 & 80 \\
\hline PI 176924 & Turkey & 93 & 86 \\
\hline PI 390261 & Japan & 45 & 133 \\
\hline PI 339243 & Turkey & 68 & 110 \\
\hline PI 390240 & Japan & 48 & 151 \\
\hline PI 204690 & Turkey & 101 & 77 \\
\hline PI 255937 & Netherlands & 75 & 99 \\
\hline PI 163213 & India & 89 & 89 \\
\hline PI 390262 & Japan & 101 & 70 \\
\hline PI 390264 & Japan & 65 & 112 \\
\hline PI 306180 & Poland & 67 & 110 \\
\hline PI 109275 & Turkey & 84 & 95 \\
\hline PI 368549 & Yugoslavia & --- & 89 \\
\hline PI 171608 & Turkey & 97 & 80 \\
\hline PI 209067 & U.S.-Ohio & 88 & --- \\
\hline PI 357845 & Yugoslavia & 91 & 85 \\
\hline PI 458856 & USSR & 92 & 83 \\
\hline PI 271334 & India & 96 & 76 \\
\hline PI 197085 & India & 72 & 104 \\
\hline PI 422169 & Czechoslovakia & 63 & 113 \\
\hline PI 357836 & Yugoslavia & 90 & 87 \\
\hline PI 422200 & Czechoslovakia & 68 & 101 \\
\hline PI 209066 & U.S.-Ohio & 49 & 125 \\
\hline PI 176523 & Turkey & 90 & 84 \\
\hline PI 288991 & Hungary & 85 & 89 \\
\hline PI 109483 & Turkey & 73 & 101 \\
\hline PI 357834 & Yugoslavia & 80 & 94 \\
\hline PI 288332 & India & 78 & 95 \\
\hline PI 432879 & P.R. China & 64 & 110 \\
\hline PI 177361 & Turkey & 112 & 70 \\
\hline PI 264227 & France & 94 & 79 \\
\hline PI 249562 & Thailand & 51 & 123 \\
\hline PI 250147 & Pakistan & 96 & 77 \\
\hline PI 218199 & Lebanon & 90 & 83 \\
\hline PI 321008 & Taiwan & 97 & 79 \\
\hline PI 391569 & P.R. China & 95 & 81 \\
\hline PI 173893 & India & 73 & 95 \\
\hline PI 178887 & Turkey & 89 & 82 \\
\hline PI 296120 & Egypt & 97 & 75 \\
\hline PI 357840 & Yugoslavia & 62 & 101 \\
\hline PI 220169 & Afghanistan & 80 & 92 \\
\hline PI 338236 & Turkey & 71 & 100 \\
\hline PI 261608 & Spain & 84 & 87 \\
\hline PI 197087 & India & 72 & 99 \\
\hline PI 218036 & Iran & 100 & 63 \\
\hline PI 390246 & Japan & 54 & 132 \\
\hline PI 181752 & Syria & 108 & 62 \\
\hline PI 314425 & USSR & 89 & 78 \\
\hline PI 211986 & Iran & 84 & 85 \\
\hline SMR 58 & Check $^{\mathrm{x}}$ & 80 & 89 \\
\hline PI 223841 & Philippines & 91 & 77 \\
\hline PI 478366 & P.R. China & 92 & 72 \\
\hline Gy 14 & Check $^{\mathrm{x}}$ & 86 & 81 \\
\hline PI 357833 & Yugoslavia & 62 & 106 \\
\hline Sumter & Check $^{\mathrm{x}}$ & 67 & 100 \\
\hline
\end{tabular}

Table 3. Continued.

\begin{tabular}{|c|c|c|c|}
\hline \multirow[b]{2}{*}{ Cultigen name } & \multirow[b]{2}{*}{ Seed source ${ }^{z}$} & \multicolumn{2}{|c|}{ Fruit yield $^{y}$} \\
\hline & & North Carolina $^{z}$ & Iowa $^{z}$ \\
\hline Everbearing & NSSL & 70 & 97 \\
\hline PI $357837^{\circ}$ & Yugoslavia & 89 & 78 \\
\hline PI 285610 & Poland & 86 & 81 \\
\hline PI 368557 & Yugoslavia & 83 & --- \\
\hline PI 419078 & P.R. China & 72 & 101 \\
\hline PI 137851 & Iran & 83 & --- \\
\hline PI 357849 & Yugoslavia & 72 & 95 \\
\hline PI 458853 & USSR & 41 & 125 \\
\hline PI 432863 & P.R. China & 80 & 87 \\
\hline PI 211967 & Iran & 79 & 87 \\
\hline PI 269482 & Pakistan & 85 & 80 \\
\hline PI 432890 & P.R. China & 91 & 66 \\
\hline PI 432851 & P.R. China & 60 & 116 \\
\hline PI 432893 & P.R. China & 86 & 79 \\
\hline PI 436608 & P.R. China & 86 & 77 \\
\hline PI 358814 & Malaysia & 74 & 104 \\
\hline Muronium & NSSL & 78 & 86 \\
\hline PI 368560 & Yugoslavia & 68 & 108 \\
\hline PI 175950 & Turkey & 70 & 93 \\
\hline PI 202801 & Syria & 81 & 81 \\
\hline PI 267742 & P.R. China & 70 & 92 \\
\hline PI 175692 & Turkey & 112 & 50 \\
\hline Delicatesse & NSSL & 67 & 95 \\
\hline PI 390954 & USSR & 79 & 83 \\
\hline PI 207476 & Afghanistan & 90 & 70 \\
\hline PI 176526 & Turkey & 91 & 73 \\
\hline PI 357832 & Yugoslavia & 110 & 51 \\
\hline Davis Perfect & NSSL & --- & 80 \\
\hline PI 372587 & Netherlands & 88 & 67 \\
\hline PI 432888 & P.R. China & 54 & 97 \\
\hline PI 212059 & Greece & 52 & 107 \\
\hline PI 432859 & P.R. China & 98 & 53 \\
\hline PI 481616 & Bhutan & --- & 79 \\
\hline PI 263078 & USSR & 58 & 100 \\
\hline Addis & Check $^{\mathrm{x}}$ & 50 & 108 \\
\hline PI 169334 & Turkey & 84 & 73 \\
\hline PI 206953 & Turkey & 88 & 69 \\
\hline PI 422196 & Czechoslovakia & 76 & 81 \\
\hline White Wonder & NSSL & 56 & 100 \\
\hline PI 390245 & Japan & 47 & 109 \\
\hline PI 279466 & Japan & 84 & 72 \\
\hline Delcrow & NSSL & 73 & 84 \\
\hline MR 200 & NSSL & 86 & 65 \\
\hline PI 357831 & Yugoslavia & 62 & 92 \\
\hline PI 344438 & Iran & 70 & 83 \\
\hline Shogoin & NSSL & 79 & 72 \\
\hline PI 432895 & P.R. China & 102 & 59 \\
\hline PI 197088 & India & 69 & 83 \\
\hline Redlands & Australia & 47 & 106 \\
\hline PI 390257 & Japan & 72 & 82 \\
\hline PI 390266 & Japan & 40 & 99 \\
\hline PI 163223 & India & 70 & 81 \\
\hline PI 390260 & Japan & 56 & 93 \\
\hline PI 344440 & Iran & 62 & 93 \\
\hline PI 481614 & Bhutan & & 74 \\
\hline PI 255934 & Netherlands & 77 & 72 \\
\hline PI 357860 & Yugoslavia & 73 & 74 \\
\hline PI 344352 & Turkey & 66 & 80 \\
\hline PI 390244 & Japan & 56 & 98 \\
\hline PI 357830 & Yugoslavia & 49 & 97 \\
\hline PI 222720 & Iran & 63 & 81 \\
\hline PI 357864 & Yugoslavia & 3 & 95 \\
\hline Wautoma & Check $^{\mathrm{x}}$ & 42 & 97 \\
\hline PI 296121 & Egypt & 52 & 92 \\
\hline PI 357854 & Yugoslavia & 64 & 80 \\
\hline PI 263049 & USSR & 56 & 87 \\
\hline PI 418989 & P.R. China & 19 & 97 \\
\hline PI 368552 & Yugoslavia & 22 & 119 \\
\hline PI 368555 & Yugoslavia & 32 & 129 \\
\hline WS Davis Perfect & NSSL & 72 & 65 \\
\hline PI 432855 & P.R. China & 90 & 49 \\
\hline PI 211728 & Afghanistan & 75 & 65 \\
\hline PI 214049 & India & 65 & 74 \\
\hline & & & \\
\hline
\end{tabular}


Retest. Eight high-yielding and four lowyielding cultigens were retested along with four checks at the Horticultural Crops Experiment Station, Clinton, in 1989. In the retest, the cultigens per se were tested rather than their hybrids with Gy 14. Seeds were planted on raised, shaped beds $1.5 \mathrm{~m}$ apart. Plots were $1.2 \mathrm{~m}$ long $\times 1.5 \mathrm{~m}$ wide with $1.2-\mathrm{m}$ alleys at each end. The guard rows were planted on the outside of the field, and at the end of each row using 'Sumter' as a monoecious pollinizer.

The experiment was a randomized complete-block design with 16 cultigens and eight replications. Plots were harvested six times (twice each week for 3 weeks) during Spring 1989, number and weight of marketable and cull fruit were recorded. Plant stand and flower count data were taken 3 and 5 weeks after planting. Number of pistillate and staminate nodes at the first five nodes of five plants were counted on each plot. Data were analyzed using the GLM procedure of SAS.

\section{Results and Discussion}

The cultigens produced hybrids differing significantly for all the traits evaluated (Table 1). We detected significant differences between the two locations for total yield, corrected total yield, and percentage of early fruit. Cultigen $\times$ location interaction was significant $(P<0.01)$ for all the traits that were evaluated at both locations. The location effect was removed by standardizing the data to form standardized total and standardized corrected total yield.

The traits that were most reliable over both locations were total yield, corrected total yield, standardized total yield, and standardized cor- rected total yield. Correction for plant stand improved the data by reducing the $\mathrm{CV}$ relative to that for corrected total yield. Therefore, data are presented as the standardized corrected total yield for all cultigens over both locations. In the analysis of variance, mean squares for location and cultigen by location interaction were highly significant, so standardized corrected total yields for cultigens were reported for each location, rather than the mean standardized corrected total yields over both locations. This was consistent with the findings of Wehner (1987b) who reported that genotype and environment were important sources of variation for yield in once-over harvest trials.

The cultigen mean square was significant for all traits evaluated, indicating that some cultigens yielded significantly more than others. The corrected yield had a better (smaller) $\mathrm{CV}$, and a better (larger) range/LSD than did total yield. Therefore, we decided to choose corrected total yield over total yield (Table 1). An additional benefit was that corrected total yield took into consideration the differences in plant stand. We decided to present yield as standardized corrected total because it had a better (lower) CV, better (lower) LSD, and a better (higher) range/LSD than did corrected total yield. The other advantage of using standardized corrected total yield was that it permitted comparison of cultigens even when some were missing from some replications.

We observed highly significant differences for location and location by cultigen interaction for percentage of early fruit (Table 1). The percentage of early fruit for Iowa had a mean of 100 , a range of 30 , and a range/LSD of 3 . The percentage of early fruit for North Carolina had a mean of 15 , a range of 63 , and range/LSD of 3. Marketable yield was recorded only in North Carolina.

In general, correlations between locations for the yield traits were low (Table 2); $r$ values for total yield, standardized total yield, corrected total yield, and standardized corrected total yield were $0.33,0.34,0.21$, and 0.23 , respectively. Although the correlations for standardized corrected total yield were slightly lower than those for total and standardized total yield, standardized corrected yield was more useful because it was corrected for both plant stand and location.

Standardized corrected total yield, which was the only trait reported for the 761 gynoecious hybrids, differed significantly in North Carolina and Iowa (Table 3). Several PI accessions produced high-yielding hybrids with Gy 14. There were 284 cultigens whose hybrids yielded more than the standard check 'Calypso'. Thus, yield might be improved by using the high performing cultigens identified in this study. The highest yielding hybrids at both locations were produced using the following paternal parents: PI422185, PI390253, PI 175120, PI 173889, PI 267087, PI 175686, PI 178888, PI 385967, PI 458851, and PI 171601. The highest yielding hybrids at North Carolina were produced using the following paternal parents: PI 175120, PI 390253, PI 344442, 'Shamrock', PI 422185, PI 177364, PI 175686, PI 178888, PI 226461, and PI 173889. The highest yielding hybrids at Iowa were produced using the following paternal parents: PI 179678, PI 267087, PI 483344, PI 391568, PI 171610, PI 432873, PI 422174, PI 179676, PI 360939, and PI 357855.

A majority of the cultigens that were classified as high or low yielders in the germ-
Table 3. Continued.

\begin{tabular}{|c|c|c|c|}
\hline \multirow[b]{2}{*}{ Cultigen name } & \multirow[b]{2}{*}{ Seed source $^{z}$} & \multicolumn{2}{|c|}{ Fruit yieldy } \\
\hline & & North Carolina $^{z}$ & Iowa $^{z}$ \\
\hline PI 422180 & Czechoslovakia & 20 & 117 \\
\hline PI 206952 & Turkey & 103 & 33 \\
\hline Pacer & Harris-Moran & 19 & 117 \\
\hline PI 372893 & Netherlands & 66 & 70 \\
\hline PI 379280 & Yugoslavia & 72 & 64 \\
\hline PI 205181 & Turkey & 50 & 84 \\
\hline PI 344437 & Iran & 86 & 54 \\
\hline PI 432896 & P.R. China & 58 & 82 \\
\hline PI 271327 & India & 40 & 93 \\
\hline PI 357843 & Yugoslavia & 59 & 72 \\
\hline PI 481617 & Bhutan & -1 & 99 \\
\hline PI 279469 & Japan & 44 & 86 \\
\hline PI 212896 & India & 8 & 122 \\
\hline PI 422192 & Czechoslovakia & 64 & 66 \\
\hline PI 379287 & Yugoslavia & 51 & 78 \\
\hline PI 265887 & Netherlands & 62 & 67 \\
\hline PI 489753 & P.R. China & 68 & 59 \\
\hline PI 390268 & Japan & 55 & 70 \\
\hline PI 357869 & Yugoslavia & 60 & 65 \\
\hline PI 432871 & P.R. China & 49 & 88 \\
\hline PI 368553 & Yugoslavia & 34 & 89 \\
\hline PI 357848 & Yugoslavia & 51 & 72 \\
\hline PI 175111 & India & 43 & 83 \\
\hline PI 357835 & Yugoslavia & 51 & 65 \\
\hline PI 175688 & Turkey & 33 & 92 \\
\hline PI 370449 & Yugoslavia & 49 & 68 \\
\hline PI 379281 & Yugoslavia & 53 & 61 \\
\hline PI 390951 & USSR & 25 & 99 \\
\hline PI 357856 & Yugoslavia & 28 & 94 \\
\hline
\end{tabular}

Table 3. Continued.

\begin{tabular}{|c|c|c|c|}
\hline \multirow[b]{2}{*}{ Cultigen name } & \multirow[b]{2}{*}{ Seed source ${ }^{z}$} & \multicolumn{2}{|c|}{ Fruit yield $^{\mathrm{y}}$} \\
\hline & & North Carolina $^{z}$ & Iowa ${ }^{z}$ \\
\hline PI 183127 & India & 17 & 92 \\
\hline PI 458855 & USSR & 57 & 46 \\
\hline PI 370450 & Yugoslavia & 33 & 80 \\
\hline Magnolia & NSSL & 51 & --- \\
\hline PI 379283 & Yugoslavia & 50 & --- \\
\hline M 27 & Check $^{\mathrm{x}}$ & 68 & 17 \\
\hline PI 370447 & Yugoslavia & 30 & 53 \\
\hline PI 183056 & India & 3 & 104 \\
\hline PI 379284 & Yugoslavia & 34 & 55 \\
\hline PI 379282 & Yugoslavia & 42 & 42 \\
\hline PI 432874 & P.R. China & 34 & 49 \\
\hline Stono & NSSL & --- & 38 \\
\hline WI 2757 & Check $^{\mathrm{x}}$ & 14 & 67 \\
\hline Marketmore 76 & Check $^{\mathrm{x}}$ & 18 & 49 \\
\hline Cubit & NSSL & 11 & 45 \\
\hline PI 368554 & Yugoslavia & 18 & 36 \\
\hline PI 357853 & Yugoslavia & 9 & 56 \\
\hline PI 368559 & Yugoslavia & 12 & 57 \\
\hline Mean & & 100 & 100 \\
\hline $\operatorname{LSD}(5 \%)$ & & 47 & 27 \\
\hline $\mathrm{CV}$ & & 29 & 17 \\
\hline Range & & 183 & 172 \\
\hline Range/LsD & & 4 & 6 \\
\hline
\end{tabular}

${ }^{\mathrm{z}}$ Some countries listed as the origin of some accessions (e.g., Czechoslovakia, USSR, Yugoslavia) now no longer exist as political units.

${ }^{y}$ Data are means of three replications.

${ }^{x}$ For the checks, yield was measured per se, rather than as combining ability with Gy 14 
Table 4. Fruit yield traits ${ }^{2}$ for the 17 cultigens retested at Clinton, N.C. (cultigens ranked by total fruit number).

\begin{tabular}{|c|c|c|c|c|c|c|c|c|}
\hline \multirow[b]{2}{*}{ Cultigen } & \multirow[b]{2}{*}{$\begin{array}{c}\text { Seed } \\
\text { source }\end{array}$} & \multirow[b]{2}{*}{$\begin{array}{l}\text { Yield } \\
\text { class }\end{array}$} & \multicolumn{2}{|c|}{ Total fruit/ha } & \multicolumn{2}{|l|}{ Fruit } & \multicolumn{2}{|c|}{ Nodes $^{y}$} \\
\hline & & & $\begin{array}{l}\text { No. } \\
(1000 s)\end{array}$ & $\begin{array}{c}\mathrm{Wt} \\
(\mathrm{Mg})\end{array}$ & $\begin{array}{c}\text { Marketable } \\
(\%)\end{array}$ & $\begin{array}{l}\text { Cull } \\
(\%)\end{array}$ & $\begin{array}{c}\text { Pistillate } \\
(\%)\end{array}$ & $\begin{array}{c}\text { Staminate } \\
(\%)\end{array}$ \\
\hline PI 169397 & Turkey & High & 915 & 67 & 84 & 16 & 6 & 89 \\
\hline Producer & NSSL & High & 662 & 58 & 91 & 9 & 29 & 66 \\
\hline Regal & Harris Moran & Check & 646 & 74 & 91 & 9 & 73 & 21 \\
\hline Poinsett 76 & Petoseed & Check & 615 & 66 & 87 & 13 & 37 & 49 \\
\hline PI 342950 & Denmark & High & 600 & 40 & 90 & 10 & 9 & 86 \\
\hline PI 175696 & Turkey & High & 586 & 74 & 83 & 17 & 7 & 90 \\
\hline Wautoma & USDA-Wisc. & Low & 512 & 45 & 95 & 5 & 1 & 92 \\
\hline PI 174172 & Turkey & High & 508 & 51 & 86 & 14 & 5 & 87 \\
\hline Wis. SMR 18 & Northrup King & Check & 484 & 54 & 87 & 13 & 8 & 85 \\
\hline PI 178888 & Turkey & High & 458 & 49 & 80 & 20 & 7 & 87 \\
\hline PI 206425 & Turkey & High & 430 & 33 & 94 & 6 & 0 & 90 \\
\hline Pacer & Harris Moran & Low & 382 & 30 & 91 & 9 & 10 & 77 \\
\hline Marketmore 76 & Asgrow Seed & Check & 355 & 21 & 89 & 11 & 1 & 89 \\
\hline PI 339250 & Turkey & High & 348 & 60 & 81 & 19 & 12 & 84 \\
\hline PI 357853 & Yugoslavia & Low & 274 & 25 & 91 & 9 & 0 & 90 \\
\hline Mean & & & 518 & 50 & 88 & 12 & 14 & 79 \\
\hline $\mathrm{CV}(\%)$ & & & 47 & 90 & 7 & 49 & 55 & 1 \\
\hline $\operatorname{LSD}(5 \%)$ & & & 244 & 17 & 6 & 6 & 8 & 10 \\
\hline Range & & & 641 & 53 & 15 & 15 & 73 & 71 \\
\hline Range/LSD & & & 3 & 3 & 3 & 3 & 9 & 7 \\
\hline
\end{tabular}

${ }^{2}$ Data are means of eight replications.

${ }^{y}$ Nodes not pistillate or staminate were barren.

plasm screening study ranked as predicted in the retest (Table 4), despite the fact that the retest was conducted using the cultigens per $s e$ and not combining ability with Gy 14 . Two exceptions were 'Wautoma' and PI 339250, which were low or high yielding when crossed with Gy 14 in the germplasm screening, but were ranked at the middle and bottom in the retest study, respectively. Thus, yield of a cultigen tested as a gynoecious hybrid (in the germplasm screening), is similar to yield of the cultigen per se with few exceptions

Once-over harvest yield might be improved using the highest yielding cultigens crossed to inbreds such as Gy 14. The top yielding cultigens could also be intercrossed to form a population from which to develop high yielding inbreds. A useful future study would be to screen the germplasm collection using ethrel to convert all cultigens to a gynoecious form for yield testing.
Miller, C.H. and G.R. Hughes. 1969. Harvest indices for pickling cucumbers in once-over harvest systems. J. Amer. Soc. Hort. Sci. 94:485487.

Nerson, H., H.S. Paris, Z. Karchi, A. Govers, M. Edelstein, and Y. Burger. 1987. Comparison of fruit-set concentration of pickling cucumbers under greenhouse and field conditions. Cucurbit Genet. Coop. Rpt. 10:2-3.

Peterson, C.E. 1975. Plant introductions in the improvement of vegetable cultivars. HortScience 10:575-579.

Schultheis, J.R. 1990. Pickling cucumbers. North Carolina State Agr. Ext. Hort. Inform. Leaflet No. 14-A.

Smith, O.S. and R.L. Lower. 1978. Field plot techniques for selecting increased once-over harvest yields in pickling cucumbers. J. Amer. Soc. Hort. Sci. 103:92-94.

Swallow, W.H. and T.C. Wehner. 1986. Optimum plot size for measurement of yield in multiple and once over harvest trials of pickling and fresh market cucumbers. Euphytica 35:493501.

Swallow, W.H. and T.C. Wehner. 1989. Optimum allocation of plots to years, seasons, locations, and replications, and its applications to onceover harvest cucumber trials. Euphytica 43:5968.

Wehner, T.C. 1989. Breeding for improved yield in cucumber. Plant Breed. Rev. (ed.) J. Janick. Vol. 6:323-359.

Wehner, T.C. 1987a. Value of 12 season-location combinations for cucumber yield trials in North Carolina. Cucurbit Genet. Coop. Rpt. 6:38-39.

rgill, B.F., D.E. Marshall, and J.H. Levin. 1975 Harvesting cucumbers mechanically. Mich. State Univ. Ext. Bul. 859

Chen, L.H., C.H. Miller, and R.S. Sowell. 1975. Simulation models for harvesting of pickling cucumbers. J. Amer. Soc. Hort. Sci. 100:176179.

Colwell, H.T.M. and J. O'Sullivan. 1981. Economics of harvest timing for once over harvesting of cucumbers. J. Amer. Soc. Hort. Sci. 106:163167.

Cramer, C.S. and T.C. Wehner. 1998. Stand correction methods for cucumber fruit yield. Cucurbit Genet. Coop. Rpt. 21:18-20.

Ells, J.E. and A.E. McSay. 1981. Yield comparisons of pickling cucumber cultivar trials for onceover harvesting. HortScience 16:187-189.

Harlan, J.R. 1975. Crops and man. Amer. Soc. Agron., Madison, Wis.

Hughes, G.R., C.W. Averre, and K.A. Sorensen. 1983. Growing pickling cucumbers in North Carolina. N.C. Agr. Ext. Serv. Bul. No. AG-315.
Wehner, T.C. 1987b. Genotype-environment interaction for cucumber yield in 23 North Carolina environments. Cucurbit Genet. Coop. Rpt. 10:25-26.

Wehner, T.C. 1986. Efficiency of three single-harvest tests for evaluation of yield in pickling cucumber. Euphytica 35:493-501.

Wehner, T.C. and C.H. Miller. 1987. Optimum plant density for multiple-harvest yield of determinate cucumbers. Cucurbit Genet. Coop. Rpt. 10:29-30.

Wehner, T.C. and C.H. Miller. 1984. Efficiency of single-harvest methods for measurement of yield in fresh-market cucumbers. J. Amer. Soc. Hort. Sci. 109:659-664.

Wehner, T.C., T.J. Monaco, and A.R. Bonanno. 1984. Chemical defoliation of cucumber vines for simulation of once-over harvest in small-plot yield trials. HortScience 19:671-673. 